\title{
Behaviour of the order parameter of the simple magnet in an external field
}

\author{
M.P.Kozlovskii, I.V.Pylyuk, O.O.Prytula \\ Institute for Condensed Matter Physics \\ of the National Academy of Sciences of Ukraine, \\ 1 Svientsitskii Str., 79011 Lviv, Ukraine
}

Received July 18, 2005, in final form October 31, 2005

\begin{abstract}
The effect of a homogeneous external field on the three-dimensional uniaxial magnet behaviour near the critical point is investigated within the framework of the nonperturbative collective variables method using the $\rho^{4}$ model. The research is carried out for the low-temperature region. The analytic explicit expressions for the free energy, average spin moment and susceptibility are obtained for weak and strong fields in comparison with the field value belonging to the pseudocritical line. The calculations are performed on the microscopic level without any adjusting parameters. It is established that the long-wave fluctuations of the order parameter play a crucial role in forming a crossover between the temperature-dependence and fielddependence critical behaviour of the system.
\end{abstract}

Key words: critical point, order parameter, Ising model

PACS: $05.50 .+q, 05.70 . \mathrm{Ce}, 64.60 . \mathrm{Fr}, 75.10 . \mathrm{Hk}$

\section{Introduction}

We propose an approach for describing the critical behaviour of a three-dimensional (3D) uniaxial magnet. Despite the variety of investigations, the problem has not been solved exactly so far [1]. Another reason of studying this model is its possible application to the study of nonmagnetic systems, such as binary alloys, simple fluids, micellar systems and so on. The second order phase transitions in the systems belonging to the 3D Ising universality class are also expected in highenergy physics [2]. Most investigations are devoted to the calculations of universal characteristics of the system, particularly, critical exponents and amplitude ratios of thermodynamic functions.

The description of the system taking into account the effect of the external field appears to be a more complicated problem. It is well known that the presence of the field causes the smearing of second order phase transition. In the vicinity of the critical point, the singularities of some thermodynamic functions transform into the 
maxima at fixed values of the field and temperature. These values correspond to the case when the effects of the field and temperature on the critical behaviour of the system are equivalent. Therefore, the simple series expansions in the scaling variable are not valid in this region, since this variable is of the order of unity [3]. In this region, the relations of thermal properties of the spin models to the properties of the clusters of spins in geometrical terms [4] are of great interest.

Early investigations regarding the 3D Ising model in the external field are reported in [5]. It is performed along the critical "isoterm" $\left(T=T_{\mathrm{c}}\right)$ using the series expansion technique. In [6], the description of the system is carried out employing the transfer matrix method. The asymptotic form of the low-temperature free energy for this model was obtained. However, this description is valid only for low temperatures. The results presented in [7] were obtained using the quantum field theory and renormalization group (RG) technique. All calculations are based on the perturbative expansion at fixed $d=3$ dimension. The equation of state is determined numerically using the parametric representation. The main difficulty in using this approach is to extrapolate the field theory results from $T>T_{\mathrm{c}}$ to $T<T_{\mathrm{c}}$ region ( $T_{\mathrm{c}}$ is the critical temperature). To solve this problem, it is necessary to proceed with analytical continuation in the complex $\tau$-plane $(\tau$ is the reduced temperature $\left.\left(\tau=\left(T-T_{\mathrm{c}}\right) / T_{\mathrm{c}}\right)\right)$. Consequently, the calculations of thermodynamic functions and their ratios of amplitude are complex. In order to determine the equation of state in parametric representation, one should employ some adjusting parameters. This is another disadvantage of this approach. The equation of state in parametric representation can be also obtained using the high-temperature expansion results [8]. There are other investigations devoted to this problem, that are performed by numerical methods [9-12].

In this article, the description of the 3D Ising-like magnet near the critical point in the external field by the nonperturbative collective variables (CV) method $[13,14]$ is presented for the case of $T<T_{\mathrm{c}}$. Using the transition from the spin variables to the collective variables, which play the role of the modes of spin density oscillations, one can calculate both universal and nonuniversal characteristics of the system. Particularly, we determine the explicit expressions for free energy, average spin moment and susceptibility as functions of the external field, which is introduced in the Hamiltonian from the outset. These calculations are based on the non-Gaussian quartic measure density $\left(\rho^{4}\right.$ model). Since, the investigations are preformed on the microscopic level and the interaction potential contains some microscopic parameters, one can investigate the thermodynamic characteristics of the system as functions of these parameters.

\section{Basic relations}

We consider the simplest one-component spin model on the simple cubic lattice with period $c$ in a homogeneous external field $h$. For calculations, we use the following 
approximation of Fourier transform

$$
\Phi(k)= \begin{cases}\Phi(0)\left(1-2 b^{2} k^{2}\right), & \mathbf{k} \in \mathcal{B}_{0} \\ \Phi_{0}=\Phi(0) \Phi, & \mathbf{k} \in \mathcal{B} \backslash \mathcal{B}_{0}\end{cases}
$$

where $\Phi(0)=8 \pi A(b / c)^{3}$, and regions $\mathcal{B}$ and $\mathcal{B}_{0}$ are defined as

$$
\begin{aligned}
\mathcal{B} & =\left\{\mathbf{k}=\left(k_{x}, k_{y}, k_{z}\right) \mid k_{i}=-\frac{\pi}{c}+\frac{2 \pi}{c} \frac{n_{i}}{N_{i}} ; n_{i}=1,2, \ldots, N_{i}, i=x, y, z\right\}, \\
\mathcal{B}_{0} & =\left\{\mathbf{k}=\left(k_{x}, k_{y}, k_{z}\right) \mid k_{i}=-\frac{\pi}{c_{0}}+\frac{2 \pi}{c_{0}} \frac{n_{i}}{N_{0 i}} ; n_{i}=1,2, \ldots, N_{0 i}, i=x, y, z\right\} .
\end{aligned}
$$

The quantities $A$ and $b$ are the microscopic parameters of the interaction potential [13], $\bar{\Phi}$ is the small constant, $N_{0 i}^{3}=N_{0}, c_{0}=c s_{0}, N_{0}=s_{0}^{-d} N, d=3$ is the dimension of the space and $N$ is total number of sites. The parameter $s_{0}\left(s_{0}>1\right)$ determines the region of values $\mathbf{k} \in \mathcal{B}_{0}$, where the parabolic approximation for $\Phi(k)$ is valid. It should be noted that the interaction potential is an exponentially decreasing function of the distance $r_{\mathbf{j} \mathbf{l}}$ between particles at sites $\mathbf{j}$ and $\mathbf{l}, \Phi\left(r_{\mathbf{j} \mathbf{l}}\right)=A \exp \left(-r_{\mathbf{j} \mathbf{l}} / b\right)$. In the general form, the Fourier transform of this potential is determined as $\Phi(k)=$ $\Phi(0) /\left(1+b^{2} k^{2}\right)^{2}$. For small values of the wave vector, the parabolic approximation is effective.

The starting point of these calculations is the partition function of the $N_{0^{-}}$ multiple integral with respect to CV [15]:

$$
\begin{aligned}
Z= & Z_{0} \sqrt{2}^{N_{0}-1} e^{\tilde{a}_{0} N_{0}} \int(\mathrm{d} \rho)^{N_{0}} \exp \left[-a_{1} \sqrt{N_{0}} \rho_{0}-\frac{1}{2} \sum_{\mathbf{k} \in \mathcal{B}_{0}} \mathrm{~d}(k) \rho_{\mathbf{k}} \rho_{-\mathbf{k}}\right. \\
& -\frac{a_{3}}{3 !} N_{0}^{-1 / 2} \sum_{\substack{\mathbf{k}_{1}, \ldots, \mathbf{k}_{3} \\
\mathbf{k}_{i} \in \mathcal{B}_{0}}} \rho_{\mathbf{k}_{1}} \ldots \rho_{\mathbf{k}_{3}} \delta_{\mathbf{k}_{1}+\cdots+\mathbf{k}_{3}} \\
& \left.-\frac{a_{4}}{4 !} N_{0}^{-1} \sum_{\substack{\mathbf{k}_{1}, \ldots, \mathbf{k}_{4} \\
\mathbf{k}_{i} \in \mathcal{B}_{0}}} \rho_{\mathbf{k}_{1}} \ldots \rho_{\mathbf{k}_{4}} \delta_{\mathbf{k}_{1}+\cdots+\mathbf{k}_{4}}\right]
\end{aligned}
$$

where

$$
d(k)=\tilde{a}_{2}-\beta \Phi(0)+2 b^{2} \beta \Phi(0) k^{2}, \quad \beta=1 / k T .
$$

The appearance of the terms with odd powers of CV is caused by the presence of the field. Coefficients $Z_{0}, \tilde{a}_{0}, \tilde{a}_{2}$ and $a_{l}$ are functions of the external field [15]. The detailed procedure of calculating (4) by the step-by-step integration method is presented in $[13,14]$. It can be represented as RG transformation of the Wilson type with renormalization parameter $s(s>1)$. After performing $n_{p}+1$ iterations, as a result, we obtain the partition function in the following form:

$$
Z=Z_{0} Q_{0} Q_{1} \ldots Q_{n_{p}} j_{n_{p}+1}\left[Q\left(P^{\left(n_{p}\right)}\right)\right]^{N_{n_{p}+1}} I_{n_{p}+1}
$$

The partial partition functions are written as

$$
Q_{n}=\left[Q\left(P^{(n-1)}\right) Q\left(d_{n}\right)\right]^{N_{n}}
$$


Here $j_{n_{p}+1}=\sqrt{2}^{N_{n_{p}+1}-1}$, and general expressions for $Q\left(P^{(n-1)}\right)$ and $Q\left(d_{n}\right)$ are given in [16]. The quantity $I_{n_{p}+1}$ from (6) is presented as [15]

$$
\begin{aligned}
I_{n_{p}+1}= & \int(\mathrm{d} \rho)^{N_{n_{p}}+1} \exp \left[-\tilde{a}_{1}^{\left(n_{p}+1\right)} N_{n_{p}+1}^{1 / 2} \rho_{0}-\frac{1}{2} \sum_{\mathbf{k} \in \mathcal{B}_{p}} d_{n_{p}+1}(k) \rho_{\mathbf{k}} \rho_{-\mathbf{k}}\right. \\
& -\frac{1}{3 !} a_{3}^{\left(n_{p}+1\right)} N_{n_{p}+1}^{-1 / 2} \sum_{\substack{\mathbf{k}_{1}, \ldots \mathbf{k}_{3} \\
\mathbf{k}_{i} \in \mathcal{B}_{p}}} \rho_{\mathbf{k}_{1}} \ldots \rho_{\mathbf{k}_{3}} \delta_{\mathbf{k}_{1}+\cdots+\mathbf{k}_{3}} \\
& \left.-\frac{1}{4 !} a_{4}^{\left(n_{p}+1\right)} N_{n_{p}+1}^{-1} \sum_{\substack{\mathbf{k}_{1}, \ldots, \mathbf{k}_{4} \\
\mathbf{k}_{i} \in \mathcal{B}_{p}}} \rho_{\mathbf{k}_{1}} \ldots \rho_{\mathbf{k}_{4}} \delta_{\mathbf{k}_{1}+\cdots+\mathbf{k}_{4}}\right] .
\end{aligned}
$$

The region $\mathcal{B}_{p} \equiv \mathcal{B}_{n_{p}+1}$ has the form similar to (3) for the lattice with period $c_{n_{p}+1}=c_{0} s^{n_{p}+1}$, where $s$ is the RG parameter $(s>1)$. The coefficients in $(7)$ are expressed through initial coefficients using the recurrence relations (RR). Passing on from quantities $a_{l}^{(n)}$ to $w_{n}, r_{n}, v_{n}$ and $u_{n}$ by relations

$$
\begin{array}{ll}
\tilde{a}_{1}^{(n)}=s^{-n} w_{n}, & d_{n}(0)=s^{-2 n} r_{n}, \\
a_{3}^{(n)}=s^{-3 n} v_{n}, & a_{4}^{(n)}=s^{-4 n} u_{n},
\end{array}
$$

new quantities $w_{n}, r_{n}, v_{n}$ and $u_{n}$ are defined by solutions of RR linearized near the fixed point $\left(w^{*}=0, r^{*}=-f_{0} \beta \Phi(0), v^{*}=0, u^{*}=\varphi_{0}(\beta \Phi(0))^{2}\right)$. In the case of $T<T_{\mathrm{c}}$, they have the form

$$
\begin{aligned}
& w_{n}=-c_{h 1} h^{\prime} E_{1}^{n}-c_{h 2} h^{\prime} T_{13}^{(0)}\left(\varphi_{0}^{1 / 2} \beta \Phi(0)\right)^{-1} E_{3}^{n}, \\
& r_{n}=r^{*}-c_{k 1}^{(0)} \beta \Phi(0) \tau_{1} E_{2}^{n}+c_{k 2} T_{24}^{(0)}\left(\varphi_{0}^{1 / 2} \beta \Phi(0)\right)^{-1} E_{4}^{n}, \\
& v_{n}=-c_{h 2} h^{\prime} E_{3}^{n} \\
& u_{n}=u^{*}-c_{k 1}^{(0)}(\beta \Phi(0))^{2} T_{42}^{(0)} \varphi_{0}^{1 / 2} \tau_{1} E_{2}^{n}+c_{k 2} E_{4}^{n} .
\end{aligned}
$$

Here $h^{\prime}=\beta h$ is the reduced external field, $\tau_{1}=-\tau, E_{l}$ are eigenvalues of the RG transformation matrix $\left(E_{1}=20.977, E_{2}=7.374, E_{3}=1.838\right.$ and $\left.E_{4}=0.397\right)$ [15]. Other quantities are some coefficients, which do not depend on the field and temperature [16]. The condition of small deviations of quantities $w_{n}, r_{n}, v_{n}$ and $u_{n}$ from the fixed point defines some value $n=n_{p}$, at which the system leaves the critical regime region (the exit point). Taking into account the eigenvalues $E_{l}$, one can see, that deviations are mainly formed in the first two equations of (9) by temperature $\tau$ and field $h$ variables. In order to obtain explicit dependences for thermodynamic functions, we use the approximation, that the exit point $n_{p}$ is only a function of one of the variables $h^{\prime}$ and $\tau$. One chooses the variable, which has a stronger effect on the critical behaviour than the other one. Hence, there are two cases: the weak field region is determined by the equality

$$
n_{p}=\mu_{\tau}=-\frac{\ln \tilde{\tau}_{1}}{\ln E_{2}}-1
$$


where the quantity $\mu_{\tau}$ defines the exit point by the temperature value, and for the strong field region we have

$$
n_{p}=n_{h}=-\frac{\ln \tilde{h}}{\ln E_{1}}-1,
$$

where $n_{h}$ is the exit point controlled by the field. Here $\tilde{h}=h^{\prime} / f_{0}$ and $\tilde{\tau}_{1}=c_{k 1}^{(0)} \tau_{1} / f_{0}$. The equality $\mu_{\tau}=n_{h}$ corresponds to the case when the effects of the temperature and the field on the critical behaviour of the system are equivalent. In the fieldtemperature plane in double logarithmic scale of the plot, this case is represented by the so-called pseudocritical line

$$
\tilde{h}_{\mathrm{c}}=\tilde{\tau}_{1}^{\beta \delta}
$$

where the quantities $\beta$ and $\delta$ are the critical exponents.

\section{Free energy of the system and equation of state}

According to the formula (6), it is convenient to write down the free energy in the form: [15]

$$
F_{e}=F_{0}+F_{\mathrm{CR}}+F_{\mathrm{TR}}+F_{I} .
$$

The term $F_{0}$ corresponds to the contribution from the noninteracting spins (in the case of $\bar{\Phi}=0$ ). It has the following form

$$
F_{0}=-k T N\left(\ln 2+\ln \cosh h^{\prime}+\frac{1}{2} \beta \Phi(0) \bar{\Phi}\right) .
$$

The term $F_{\mathrm{CR}}$ is the result of $\mathrm{RG}$ transformations and represents the contribution from the short-wave oscillation modes. The expression for $F_{\mathrm{CR}}$ is written as

$$
\begin{aligned}
F_{\mathrm{CR}}= & -k T N_{0}\left[e_{0 p}-e_{1 p} \tilde{\tau}_{1}+e_{2 p} \tilde{\tau}_{1}^{2}+e_{3 p} \tilde{h}^{2}\right. \\
& \left.+\left(-F_{10}+F_{11} \tilde{\tau}_{1} E_{2}^{n_{p}+1}-F_{12} \tilde{\tau}_{1}^{2} E_{2}^{2\left(n_{p}+1\right)}\right) s^{-3\left(n_{p}+1\right)}\right] .
\end{aligned}
$$

For the quantity $F_{\mathrm{TR}}$, we have

$$
F_{\mathrm{TR}}=-k T N_{0} s^{-3\left(n_{p}+1\right)}\left\{f_{p 1 c}-n_{p} \ln s-f_{p 11 c} \tilde{\tau}_{1} E_{2}^{n_{p}+1}-f_{p 12 c} \tilde{\tau}_{1}^{2} E_{2}^{2\left(n_{p}+1\right)}\right\} .
$$

This is the free energy of the regime, which corresponds to the transition from shortwave to long-wave oscillation modes of the order parameter. In expressions (15) and (16), the quantities $F_{1 l}, e_{l p}, f_{p 1 c}$ and $f_{p 1 l c}$ are independent of the field [16].

The term from (13), which represents the contribution from the long-wave fluctuations, has the form

$$
F_{I}=-k T \ln I_{n_{p}+1}
$$


The quantity $I_{n_{p}+1}$ is calculated using the series of substitutions of variables. The first of them is

$$
\rho_{\mathbf{k}}=\eta_{\mathbf{k}}+\sigma_{h} \sqrt{N} \delta_{\mathbf{k}}
$$

Thus, we obtain

$$
\begin{aligned}
I_{n_{p}+1}= & \exp \left[E_{0}\left(\sigma_{h}\right)\right] \int(\mathrm{d} \eta)^{N_{n_{p}+1}} \exp \left[A_{0} \sqrt{N} \eta_{0}-\frac{1}{2} \sum_{\mathbf{k} \in \mathcal{B}_{n_{p}+1}} d_{h}(k) \eta_{\mathbf{k}} \eta_{-\mathbf{k}}\right. \\
& -\frac{1}{3 !} b_{h} N_{n_{p}+1}^{-1 / 2} \sum_{\substack{\mathbf{k}_{1}, \ldots, \mathbf{k}_{3} \\
\mathbf{k}_{i} \in \mathcal{B}_{n_{p}+1}}} \eta_{\mathbf{k}_{1}} \ldots \eta_{\mathbf{k}_{3}} \delta_{\mathbf{k}_{1}+\cdots+\mathbf{k}_{3}} \\
& \left.-\frac{1}{4 !} a_{h} N_{n_{p}+1}^{-1} \sum_{\substack{\mathbf{k}_{1}, \ldots, \mathbf{k}_{4} \\
\mathbf{k}_{i} \in \mathcal{B}_{n_{p}+1}}} \eta_{\mathbf{k}_{1}} \ldots \eta_{\mathbf{k}_{4}} \delta_{\mathbf{k}_{1}+\cdots+\mathbf{k}_{4}}\right]
\end{aligned}
$$

Here

$$
\begin{aligned}
A_{0} & =a_{1 m} \tilde{h}-d_{n_{p}+1}(0) \sigma_{h}-\frac{1}{6} a_{4}^{\left(n_{p}+1\right)} \sigma_{h}^{3} \frac{N}{N_{n_{p}+1}} \\
d_{h}(k) & =d_{h}(0)+2 \beta \Phi(0) b^{2} k^{2}, \quad d_{h}(0)=d_{n_{p}+1}(0)+\frac{1}{2} a_{4}^{\left(n_{p}+1\right)} \sigma_{h}^{2} \frac{N}{N_{n_{p}+1}} \\
b_{h} & =\sigma_{h} a_{4}^{\left(n_{p}+1\right)}\left(\frac{N}{N_{n_{p}+1}}\right)^{\frac{1}{2}}, \quad a_{h}=a_{4}^{\left(n_{p}+1\right)}
\end{aligned}
$$

and

$$
E_{0}\left(\sigma_{h}\right)=N\left[a_{1 m} \sigma_{h} \tilde{h}-\frac{1}{2} d_{n_{p}+1}(0) \sigma_{h}^{2}-\frac{a_{4}^{\left(n_{p}+1\right)}}{24} \sigma_{h}^{4} \frac{N}{N_{n_{p}+1}}\right] .
$$

The quantity $\sigma_{h}$ can be found employing the condition $\partial E_{0}\left(\sigma_{h}\right) / \partial \sigma_{h}=0$. Performing the substitution of the variable

$$
\sigma_{h}=\sigma_{0} s^{-\frac{n_{p}+1}{2}}
$$

we arrive at the cubic equation

$$
\left[a_{1 m} \tilde{h} E_{1}^{n_{p}+1}-r_{n_{p}+1} \sigma_{0}-\frac{1}{6} u_{n_{p}+1} \sigma_{0}^{3} s_{0}^{3}\right] s^{-\frac{5}{2}\left(n_{p}+1\right)}=0 .
$$

The solution of $(22)$ is chosen using the condition of free energy minimization. It also nullifies the quantity $A_{0}$ from (19). The quadratic term in the expression of the exponent in (18) becomes positive and dominating in comparison with other terms for all $k \neq 0$. Thus, we can perform the integration in (18) with respect to the variables $\eta_{\mathbf{k}}$ except the variable $\eta_{0}$. The next step in the calculations lies in returning to the variable $\rho_{0}$ by means of

$$
\eta_{0}=\rho_{0}-\sigma_{h} \sqrt{N}
$$


The average value of the variable $\rho_{0}$ plays the role of the order parameter of the system. Therefore, performing the integration with respect to this variable, we get the main contribution to the total free energy and average spin moment. The integration is carried out performing the substitution $\rho_{0}=\rho_{h} \sqrt{N}$ and using the steepest descent method. As a result, the quantity $I_{n_{p}+1}$ assumes the following form:

$$
I_{n_{p}+1}=\prod_{\substack{\mathbf{k} \in \mathcal{B}_{n_{p}+1} \\ k \neq 0}}\left(\frac{\pi}{d_{h}(k)}\right)^{\frac{1}{2}} \sqrt{N} \int \mathrm{d} \rho_{h} \exp \left[N E_{0}\right]
$$

where

$$
E_{0}=a_{1 m} E_{1}^{n_{p}+1} s^{-\frac{5}{2}\left(n_{p}+1\right)} \tilde{h} \rho_{h}-\frac{1}{2} r_{n_{p}+1} s^{-2\left(n_{p}+1\right)} \rho_{h}^{2}-\frac{1}{4 !} s_{0}^{d} u_{n_{p}+1} s^{-\left(n_{p}+1\right)} \rho_{h}^{4} .
$$

Using the condition $\partial E_{0} / \partial \rho_{h}=0$, we find the root of the cubic equation satisfying the condition of the free energy minimization in the form

$$
\rho_{h}=\sigma_{0 h} s^{-\frac{n_{p}+1}{2}} .
$$

Thus, the quantity $I_{n_{p}+1}$ can be written as

$$
I_{n_{p}+1}=\prod_{\substack{\mathbf{k} \in \mathcal{B}_{n_{p}+1} \\ k \neq 0}}\left(\frac{\pi}{d_{h}(k)}\right)^{\frac{1}{2}} \exp \left[N E_{0}\left(\sigma_{0 h}\right)\right]
$$

where

$$
E_{0}\left(\sigma_{0 h}\right)=s^{-3\left(n_{p}+1\right)}\left[a_{1 m} E_{1}^{n_{p}+1} \tilde{h} \sigma_{0 h}-\frac{1}{2} r_{n_{p}+1} \sigma_{0 h}^{2}-\frac{1}{4 !} s_{0}^{d} u_{n_{p}+1} \sigma_{0 h}^{4}\right]
$$

As one can see, this quantity is the function of the variable $\rho_{0}$, whose average value is the order parameter as it was mentioned above. Thus, the expression (27) is the microscopic analog of the Landau free energy as well as the relation (25) is the equation of state.

Taking the logarithm, transiting to the spherical Brillouin zone and integrating with respect to $k$ in the expression (26), we arrive at the formula for free energy of long-wave fluctuations:

$$
F_{I}=-k T N\left\{\left[\left(n_{p}+1\right) \ln s-\frac{1}{2} I_{0}^{\prime}+\frac{1}{2} \ln \pi\right] s_{0}^{-3}+E_{0}\left(\sigma_{0 h}\right)\right\} s^{-3\left(n_{p}+1\right)} .
$$

The quantity $I_{0}^{\prime}$ has the form

$$
I_{0}^{\prime}=\ln \left(D_{0}^{\prime}+D_{1}^{\prime}\right)-\frac{2}{3}+2 \frac{D_{0}^{\prime}}{D_{1}^{\prime}}-2\left(\frac{D_{0}^{\prime}}{D_{1}^{\prime}}\right)^{\frac{3}{2}} \arctan \left(\frac{D_{1}^{\prime}}{D_{0}^{\prime}}\right)^{\frac{1}{2}}
$$

and coefficients $D_{0}^{\prime}$ and $D_{1}^{\prime}$ are defined as

$$
D_{0}^{\prime}=r_{n_{p}+1}+\frac{1}{2} s_{0}^{3} u_{n_{p}+1} \sigma_{0 h}^{2}, \quad D_{1}^{\prime}=2 \beta \Phi(0) s_{0}^{-2}\left(\frac{\pi b}{c}\right)^{2} .
$$


Collecting the contributions from all regimes of fluctuations according to (13), we can now write down complete expressions for the free energy of the system in case of strong and weak fields. Taking into account that in the case of $n_{p}=\mu_{\tau}$ (see (10)) $s^{-3\left(\mu_{\tau}+1\right)}=\tilde{\tau}_{1}^{3 \nu}$ and $\tilde{\tau}_{1} E_{2}^{\mu_{\tau}+1}=1$, the total free energy for the weak field region takes on the form

$$
F_{e}=-k T N\left[\ln \cosh h^{\prime}+l_{0}+l_{1 \mu e} \tilde{\tau}_{1}^{3 \nu}+l_{11 \mu e} \tilde{h} \tilde{\tau}_{1}^{\frac{\nu}{2}}+l_{2} \tilde{h}^{2}-l_{3} \tilde{\tau}_{1}+l_{4} \tilde{\tau}_{1}^{2}\right],
$$

where

$$
\begin{array}{ll}
l_{0}=\ln 2+\frac{1}{2} \beta_{\mathrm{c}} \Phi(0) \bar{\Phi}+s_{0}^{-3} e_{0 p}, & \\
l_{1 \mu e}=l_{1 \mu e}^{(0)}-\frac{1}{2} r_{\mu_{\tau}+1} \sigma_{0 h}^{2}-\frac{1}{24} u_{\mu_{\tau}+1} s_{0}^{3} \sigma_{0 h}^{4}, & l_{2}=s_{0}^{-3} e_{3 p}, \\
l_{11 \mu e}=a_{1 m} \sigma_{0 h}, & l_{4}=s_{0}^{-3} e_{2 p}+\frac{1}{2} \beta_{\mathrm{c}} \Phi(0) \bar{\Phi} f_{0}^{2} /\left(c_{k 1}^{(0)}\right)^{2} . \\
l_{3}=s_{0}^{-3} e_{1 p}-\frac{1}{2} \beta_{\mathrm{c}} \Phi(0) \bar{\Phi} f_{0} / c_{k 1}^{(0)}, &
\end{array}
$$

Here $\beta_{\mathrm{c}}$ is the inverse critical temperature. The quantity $l_{1 \mu e}^{(0)}$ is obtained by the summation of coefficients proportional to $\tilde{\tau}_{1}^{3 \nu}$ from different contributions.

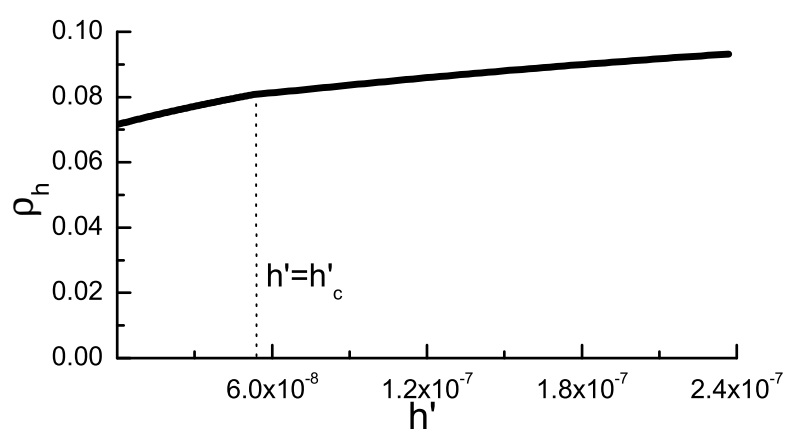

Figure 1. The contribution to the order parameter from the regime of long-wave fluctuations as a function of the field for $\tau=10^{-5}$.

According to the relation (11), we have $s^{-3\left(n_{h}+1\right)}=\tilde{h}^{\frac{6}{5}}$ and $E_{2}^{n_{h}+1}=\tilde{h}^{-\frac{1}{\beta \delta}}$. Thus, the free energy of the strong field region is written as

$$
\begin{aligned}
F_{e, h}= & -k T N\left[\ln \cosh h^{\prime}+l_{0}+l_{1 e}^{(-)} \tilde{h}^{\frac{6}{5}}-l_{11 e} \tilde{\tau}_{1} \tilde{h}^{\frac{6}{5}-\frac{1}{p_{0}}}\right. \\
& \left.+l_{12 e} \tilde{\tau}_{1}^{2} \tilde{h}^{\frac{6}{5}-\frac{2}{p_{0}}}+l_{2} \tilde{h}^{2}-l_{3} \tilde{\tau}_{1}+l_{4} \tilde{\tau}_{1}^{2}\right] .
\end{aligned}
$$

Here $p_{0}=\beta \delta$, and the coefficients satisfy the following relations:

$$
\begin{gathered}
l_{1 e}^{(-)}=E_{0, h}+\left(\ln s-\frac{1}{2} I_{0}^{\prime}+\frac{1}{2} \ln \pi+f_{p 1 c}-F_{10}\right) s_{0}^{-3}, \\
l_{11 e}=-s_{0}^{-3}\left(F_{11}-f_{p 11 c}\right), \quad l_{12 e}=-s_{0}^{-3}\left(F_{12}+f_{p 12 c}\right) .
\end{gathered}
$$


The main contribution to the order parameter (25) for weak fields $h^{\prime}<h_{\mathrm{c}}^{\prime}$ takes on the form

$$
\rho_{h}=\sigma_{0 h} \tilde{\tau}_{1}^{\frac{\nu}{2}}
$$

and for strong fields $h^{\prime}>h_{\mathrm{c}}^{\prime}$

$$
\rho_{h}=\sigma_{0 h} \tilde{h}^{\frac{1}{5}}
$$

respectively. The quantity $\sigma_{0 h}$ is the root of the cubic equation

$$
a_{1 m} \tilde{h} E_{1}^{n_{p}+1}-r_{n_{p}+1} \sigma_{0 h}-\frac{1}{6} u_{n_{p}+1} \sigma_{0 h}^{3} s_{0}^{3}=0
$$

derived from (27). The dependences (33) and (34) are shown in figure 1. As one can see, they coincide in the point $h^{\prime}=h_{\mathrm{c}}^{\prime}\left(h_{\mathrm{c}}^{\prime}=f_{0} \tilde{h}_{\mathrm{c}}\right)$. Tending to the pseudocritical line region, the coefficient $\sigma_{0 h}$ becomes essentially dependent on the field. In the point $h^{\prime}=h_{\mathrm{c}}^{\prime}$, this dependence is the most substantial. Such a dependence is related to the presence of the field in terms of the cubic equation (35). The quantity $\sigma_{0 h}$ ensures a crossover between the temperature-dependence and field-dependence critical behaviour. With the field further increasing, the dependence on the field is reduced, and $\sigma_{0 h}$ in the case of $h^{\prime} \gg h_{\mathrm{c}}^{\prime}$ becomes practically independent of the field.

The expressions (29) and (31) allow us to get other thermodynamic characteristics of the system. Particularly, the total average spin moment can be obtained using the well-known relation $\sigma=-\left(N^{-1} \cdot \mathrm{d} F / \mathrm{d} h\right)_{T}$. In the case of the small fields, the explicit expression for order parameter has the form

$$
\sigma_{e}^{(-)}=\tanh h^{\prime}+2 l_{2} f_{0}^{-2} h^{\prime}+l_{11 \mu e} f_{0}^{-1} \tilde{\tau}_{1}^{\frac{\nu}{2}}
$$

The dependence of the coefficient $l_{11 \mu e}$ on the field and temperature is responsible for the crossover between two types of the critical behaviour and for the divergence of the second order derivatives of the free energy in the critical point. When $\tilde{h}>\tilde{h}_{\mathrm{c}}$, the total order parameter can be written as follows

$$
\begin{aligned}
\sigma_{e, h}^{(-)}= & \tanh h^{\prime}+2 l_{2} f_{0}^{-2} h^{\prime}+\sigma_{0 e h} h^{\prime \frac{6}{5}}+\frac{6}{5} l_{1 e}^{(-)} f_{0}^{-\frac{6}{5}} h^{\prime \frac{1}{5}} \\
& -\sigma_{1 e h} \tilde{\tau}_{1} h^{\prime \frac{1}{5}-\frac{1}{p_{0}}}+\sigma_{2 e h} \tilde{\tau}_{1}^{2} h^{\prime \frac{1}{5}-\frac{2}{p_{0}}} .
\end{aligned}
$$

The coefficients in expressions (36), (37) and in the following expressions for the susceptibility can be obtained through the coefficients (30) and (32) by differentiating the free energy of the system with respect to the field variable for the case of $\tilde{h}<\tilde{h}_{\mathrm{c}}$ and $\tilde{h}>\tilde{h}_{\mathrm{c}}$, respectively.

In the case of $\tilde{h}<\tilde{h}_{\mathrm{c}}$, the susceptibility is defined as

$$
\chi_{e}^{(-)}=\frac{1}{k T}\left[1-\tanh ^{2} h^{\prime}+2 l_{2} f_{0}^{-2}+f_{0}^{-1} \frac{\partial l_{11 \mu e}}{\partial h} \tilde{\tau}_{1}^{\frac{\nu}{2}}\right] .
$$

When $\tilde{h}>\tilde{h}_{\mathrm{c}}$, we obtain

$$
\begin{aligned}
\chi_{e, h}^{(-)}= & \frac{1}{k T}\left[1-\tanh ^{2} h^{\prime}+2 l_{2} f_{0}^{-2}+\chi_{0 e h} h^{\prime \frac{6}{5}}+\frac{12}{5} \sigma_{0 e h} h^{\prime \frac{1}{5}}\right. \\
& \left.+\chi_{1 e h}^{(-)} h^{\prime-\frac{4}{5}}-\chi_{2 e h} \tilde{\tau}_{1} h^{\prime-\frac{4}{5}-\frac{1}{p_{0}}}+\chi_{3 e h} \tilde{\tau}_{1}^{2} h^{\prime-\frac{4}{5}-\frac{2}{p_{0}}}\right] .
\end{aligned}
$$


Due to the approximation that exit point $n_{p}$ is one-variable function, there is some disagreement between the quantities $\sigma_{e}^{(-)}, \sigma_{e, h}^{(-)}$as well as between $\chi_{e}^{(-)}$and $\chi_{e, h}^{(-)}$in the vicinity of the pseudocritical line (for example, $\chi_{e}^{(-)}=2.01 \cdot 10^{5}$ and $\chi_{e, h}^{(-)}=2.02 \cdot 10^{5}$ at $\tau=10^{-5}, h^{\prime}=h_{\mathrm{c}}^{\prime}$ ). Nevertheless, the region of disagreement is quite narrow, since the long-wave fluctuations play the major role in the critical phenomena.

\section{Conclusions}

The description of the effect of the external magnetic field on the 3D Ising-like magnet near the critical point using the CV method in the low-temperature region is presented. We get the explicit analytic expressions for the free energy, order parameter and susceptibility as functions of the field and temperature. For variable $\rho_{0}$, which plays the role of the order parameter in the CV method, the field dependence of the whole range of fields and temperatures in the vicinity of the critical point is obtained. In the proposed approach, the crossover between critical behaviour controlled by field or temperature variable is ensured mainly by the contribution from the long-wave fluctuations. Therefore, this dependence is the main contribution to the total equation of state. Since the calculations are carried out on the microscopic level, we can obtain the dependences of the nonuniversal characteristics on the microscopic parameters of the system (a lattice constant and parameters of the interaction potential). Calculations are performed within the framework of the quartic measure density, which allows one to obtain the qualitative description of the system behaviour. For more accurate estimations, it is necessary to use the $\rho^{6}$ model [17-19]. The calculations can be also extended to the classical $n$-vector magnetic model [20]. 


\section{References}

1. Pelissetto A., Vicari E., Phys. Reports, 2002, 368, 549.

2. Guttmann A.J., Phase Transitions and Critical Phenomena, edited by Domb C. and Lebowitz J., vol. 13, Academic, New York, 1989.

3. Millev Y.T., Fähnle M., Whitaker M.A.B., J. Magn. Magn. Mater., 1996, 152, 239.

4. Fortunato S., Satz H., Nucl. Phys. Proc. Suppl., 2002, 106, 890.

5. Tarko H.B., Fisher M.E., Phys. Rev. Lett., 1973, 31, 926.

6. Kochmanski M.S., J. Phys. A: Math. Gen., 1999, 32, 1251.

7. Guida R., Zinn-Justin J., Nucl. Phys. B, 1997, 489, 626.

8. Campostrini M., Pelissetto A., Rossi P., Vicari E., Phys. Rev. E, 2002, 65, 066127.

9. Blöte H.W.J., Heringa J.R., Tsypin M.M., Phys. Rev. E, 2000, 62, 77.

10. Engels J., Fromme L., Seniuch M., Nuc. Phys. B, 2003, 655, 277.

11. Tsypin M.M., Phys. Rev. Lett., 1994, 73, 2015.

12. Tsypin M.M., Phys. Rev. B, 1997, 55, 8911.

13. Yukhnovskii I.R. Phase Transitions of the Second Order. Collective Variables Method. World Scientific, Singapore, 1987.

14. Yukhnovskii I.R., Kozlovskii M.P., Pylyuk I.V. Microscopic Theory of Phase Transitions in the Three-Dimensional Systems. Eurosvit, Lviv, 2001 (in Ukrainian).

15. Kozlovskii M.P., Pylyuk I.V., Prytula O.O., Condens. Matter Phys., 2004, 7, 361.

16. Kozlovskii M.P., Pylyuk I.V., Prytula O.O. Preprint of the Institute for Condensed Matter Physics, ICMP-04-03U, Lviv, 2004, 32 p. (in Ukrainian).

17. Yukhnovskii I.R., Kozlovskii M.P., Pylyuk I.V., Phys. Rev. B, 2002, 66, 134410.

18. Yuhnovskii I.R., Pylyuk I.V., Kozlovskii M.P., J. Phys.:Condens. Matter, 2002, 14, 11701.

19. Yuhnovskii I.R., Pylyuk I.V., Kozlovskii M.P., J. Phys.:Condens. Matter, 2002, 14, 10113.

20. Usatenko Z.E., Kozlovskii M.P., Phys. Rev. B, 2000, 62, 9599. 


\section{Поведінка параметра порядку простого магнетика} в зовнішньому полі

\section{М.П.Козловський, І.В.Пилюк, О.О.Притула}

Інститут фізики конденсованих систем НАН України, 79011 Львів, вул. Свєнціцького, 1

Отримано 18 липня 2005 р., в остаточному вигляді 31 жовтня $2005 \mathrm{p}$.

В рамках непертурбативного методу колективних змінних на основі моделі $\rho^{4}$ досліджено вплив зовнішнього поля на критичну поведінку тривимірного одновісного магнетика. Дослідження проведені для низькотемпературної області. Отримані аналітичні вирази для вільної енергії, середнього спінового моменту і сприйнятливості у випадку слабких і сильних полів по відношенню до величини поля, що задовільняе рівняння псевдокритичної лінії. Розрахунки проведені на мікроскопічному рівні без застосування допоміжних параметрів. Встановлено, що вирішальну роль у формуванні переходу між температурозалежною і залежною від поля критичною поведінкою системи відіграють довгохвильові флуктуації параметра порядку.

Ключові слова: критична точка, параметр порядку, модель Ізинга

PACS: $05.50 .+q, 05.70 . \mathrm{Ce}, 64.60 . \mathrm{Fr}, 75.10 . \mathrm{Hk}$ 